\title{
La compétition partisane post-dévolution en Ecosse et au pays de Galles depuis 1999 : une divergence grandissante
}

Electoral Competition and the Devolved Party System in the British Periphery since 1999: the Growing Divergence between the Scottish and Welsh Models

\section{Gilles LEYDIER}

\section{(2) OpenEdition \\ 1 Journals}

\section{Édition électronique}

URL : http://journals.openedition.org/rfcb/1572

DOI : $10.4000 / \mathrm{rfcb} .1572$

ISSN : 2429-4373

Éditeur

CRECIB - Centre de recherche et d'études en civilisation britannique

Référence électronique

Gilles LEYDIER, «La compétition partisane post-dévolution en Ecosse et au pays de Galles depuis 1999 : une divergence grandissante », Revue Française de Civilisation Britannique [En ligne], XXII-4 | 2017, mis en ligne le 27 octobre 2017, consulté le 03 mai 2019. URL : http:// journals.openedition.org/rfcb/1572; DOI : 10.4000/rfcb.1572

Ce document a été généré automatiquement le 3 mai 2019.

\section{(c) $(1) \odot$}

Revue française de civilisation britannique est mis à disposition selon les termes de la licence Creative Commons Attribution - Pas d'Utilisation Commerciale - Pas de Modification 4.0 International. 


\title{
La compétition partisane post- dévolution en Ecosse et au pays de Galles depuis 1999 : une divergence grandissante
}

\author{
Electoral Competition and the Devolved Party System in the British Periphery \\ since 1999: the Growing Divergence between the Scottish and Welsh Models
}

Gilles LEYDIER

1 L'objet de cet article est d'analyser l'évolution de la compétition entre les partis politiques dans la périphérie britannique depuis la mise en place de la dévolution, à travers l'étude des résultats des élections aux assemblées décentralisées ${ }^{1}$.

En Ecosse comme au pays de Galles, la dévolution instaurée à la fin des années 1990 a créé de nouvelles institutions démocratiques représentatives, dotées de compétences propres s'exerçant dans un cadre territorial bien délimité, favorisant ainsi l'éclosion d'une scène politique centrée sur des enjeux locaux. Des systèmes partisans ${ }^{2}$ territorialisés ont émergé, développant leur propre dynamique tout en continuant de s'inscrire dans le cadre étatique en raison notamment de la présence majoritaire de formations politiques à vocation britannique. La comparaison de la situation dans les deux nations périphériques permet de mesurer comment l'enjeu de l'autonomie -culturelle et/ou politique- s'est traduit dans la compétition partisane depuis la mise en route du Parlement écossais et de l'Assemblée galloise en 1999.

Les deux nations périphériques ont revendiqué le principe de la dévolution, validé avec enthousiasme en Ecosse, plus timidement au pays de Galles, lors des référendums de 1997. Elles ont par ailleurs toutes deux opté pour le principe d'élections territoriales à dates fixes -avec un mandat de quatre ans pour les élus- bien avant que le Parlement britannique n'adopte cette mesure. Et elles ont choisi un même mode de scrutin original mixte dit $\mathrm{AMS}^{3}$, combinant un vote avec un système majoritaire uninominal traditionnel ( FPTP) et un vote à la proportionnelle sur des listes régionales, susceptible de favoriser le 
renouvellement de l'offre politique et une représentation partisane plus diversifiée qu'au Parlement de Westminster.

4 Au moment de la mise en route de la dévolution, L'Ecosse et le pays de Galles présentaient des systèmes partisans comparables à bien des égards, et caractérisés par un ensemble quadripolaire faisant coexister trois partis britanniques unionistes (travaillistes, conservateurs et libéraux-démocrates) et un parti nationaliste bien implanté (le SNP en Ecosse, Plaid Cymru au pays de Galles). Dans les deux cas, la compétition interpartisane était dominée par le parti travailliste, historiquement fort sur ces terres (post)industrielles et par ailleurs architecte de la dévolution institutionnelle, tandis que le débat politique s'articulait autour de deux axes principaux: l'un de type socioéconomique autour du clivage gauche-droite (avec dans les deux cas un centre de gravité situé au centre-gauche), l'autre autour des enjeux de l'identité politique et culturelle et de l'émancipation par rapport au cadre britannique, construit sur un axe nationalismeunionisme.

\section{9 : les débuts de la dévolution ou la convergence parfaite des systèmes partisans}

5 Si l'on commence l'analyse avec les premières élections aux assemblées décentralisées de mai 1999, il est frappant de constater l'extrême similitude des situations électorales entre l'Ecosse et le pays de Galles à cet instant (voir tableau 1).

Tableau 1 : Résultats obtenus aux élections au parlement écossais et à l'assemblée galloise depuis 1999 (en \% de suffrages exprimés)

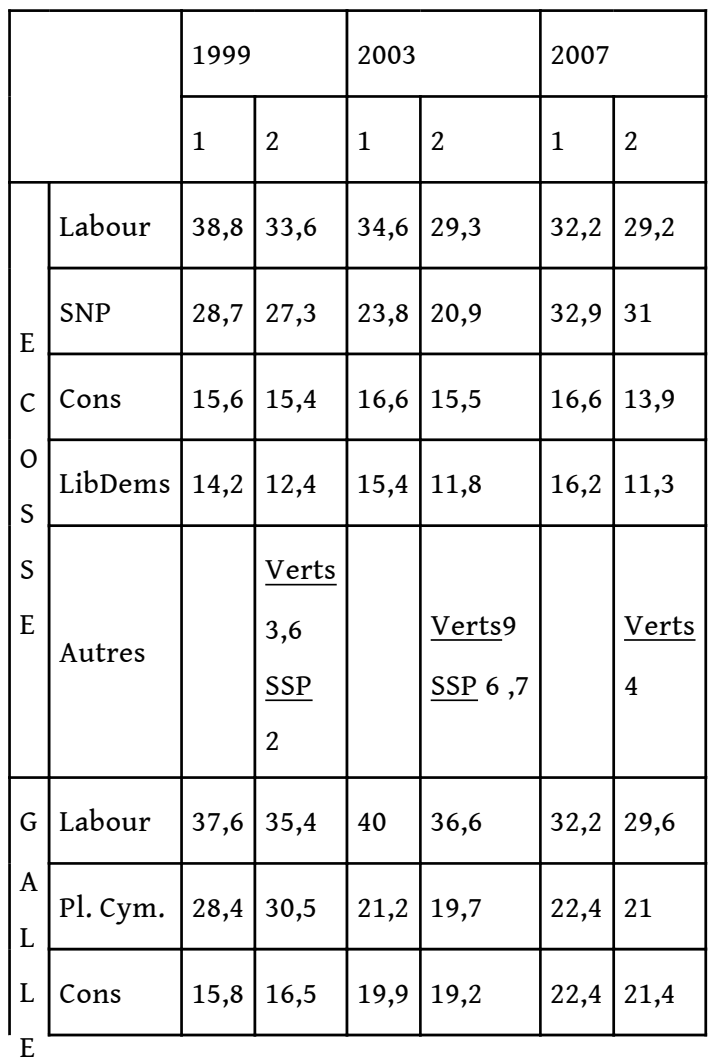




\begin{tabular}{|l|l|l|l|l|l|l|}
\hline LibDems & 13,5 & 12,5 & 14,1 & 12,7 & 14,8 & 11,7 \\
\hline & & & & & \\
Autres & & $\underline{\text { Verts }}$ & & $\frac{\text { Verts }}{3,5}$ & & 4,3 \\
& & & & $\underline{\text { UKIP }}$ & & \\
& & & & & & \\
\end{tabular}

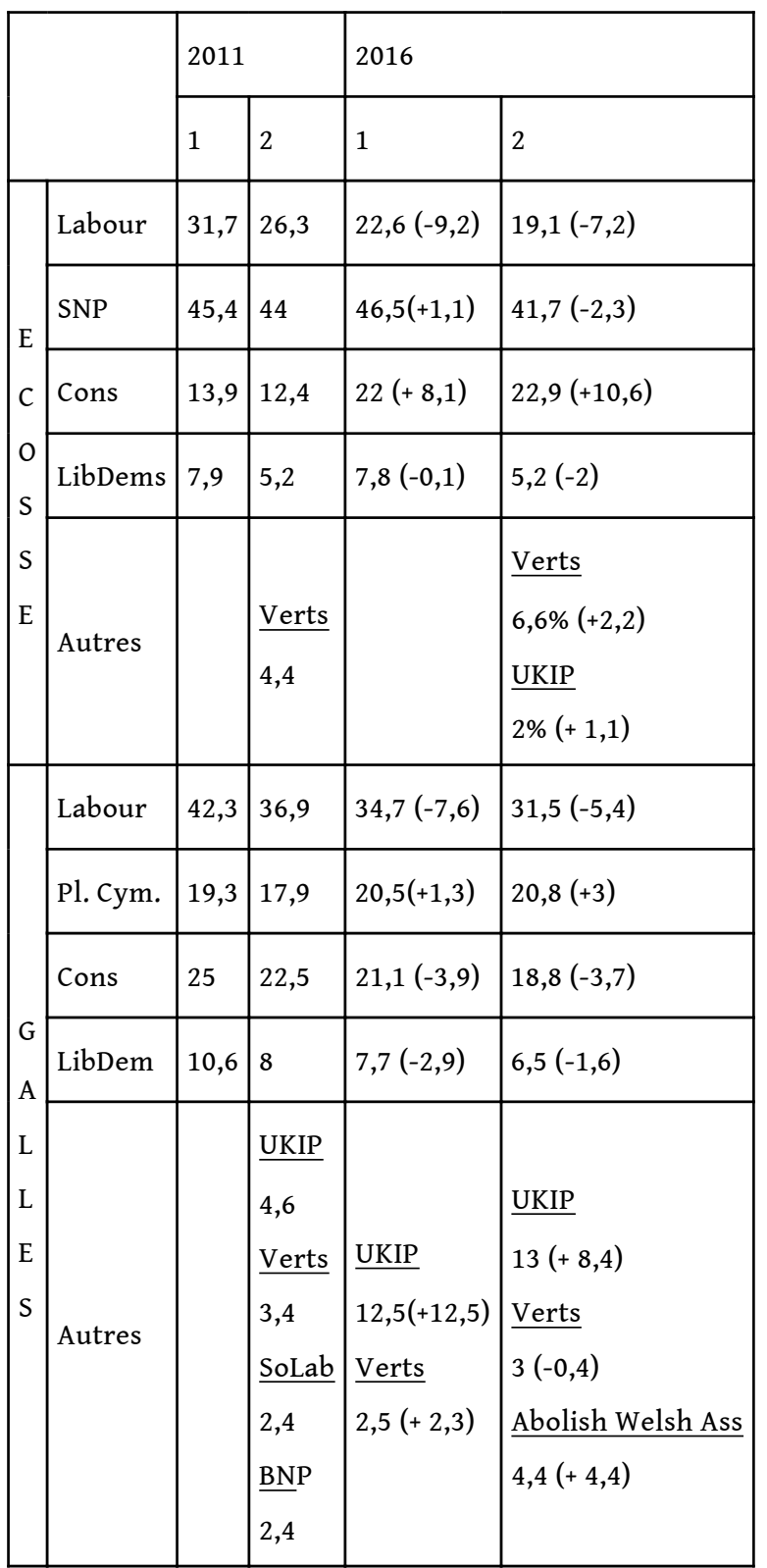

1 = vote avec le mode de scrutin FPTP (majoritaire de circonscription uninominal majoritaire à un tour) ; 2 = vote avec un scrutin à la proportionnelle de listes régionales.

Sont pris en compte les partis dont les résultats sont supérieurs ou égaux à $2 \%$.

SoLab $=$ Socialist Labour 
En Ecosse, le parti travailliste est arrivé nettement en tête dans les deux types de votes, tandis que le parti nationaliste SNP émergeait en deuxième position dans une situation de principal concurrent du Labour, alors au pouvoir à Londres. Les conservateurs et les libéraux-démocrates étaient relégués au troisième rang, avec un résultat électoral comparable, tandis que des petites formations (écologistes, gauche radicale) parvenaient à obtenir quelques sièges au parlement d'Edimbourg grâce au deuxième scrutin à la proportionnelle (voir tableau 2).

7 Si les travaillistes étaient en position de force, ce qui se traduisait par l'obtention de 53 sièges sur les 72 avec le mode de scrutin FPTP, l'existence du deuxième vote à la proportionnelle les empêchait d'obtenir la majorité absolue de la représentation à Holyrood, ce qui les conduisit à former une alliance électorale avec les libérauxdémocrates - également favorisés par le vote au scrutin FPTP- dans le cadre d'un gouvernement de coalition. Par ailleurs les conservateurs, balayés dans le cadre du premier vote, ne devaient leur représentation au Parlement écossais qu'au deuxième vote à la proportionnelle, tandis que les nationalistes du Scottish National Party ainsi que les petites formations tiraient eux-aussi bénéfice de ce mode de scrutin (voir tableau 2).

8 Tableau 2 : Résultats obtenus aux élections au parlement écossais et à l'assemblée galloise depuis 1999 (en nombre de sièges gagnés)

\begin{tabular}{|c|c|c|c|c|}
\hline & & 1999 & 2003 & 2007 \\
\hline \multirow{5}{*}{$\begin{array}{l}\text { ECOSSE } \\
129 \text { sièges } \\
= \\
73 \text { FPTP } \\
+56 \text { listes régionales }\end{array}$} & Labour & $56(53+3)$ & $50(46+4)$ & $46(37+9)$ \\
\hline & SNP & $35(7+28)$ & $27(9+18)$ & $47(21+26)$ \\
\hline & Cons & $18(0+18)$ & $18(3+15)$ & $17(4+13)$ \\
\hline & Lib-Dems & $17(12+5)$ & $17(13+4)$ & $16(11+5)$ \\
\hline & Autres & $\begin{array}{l}\underline{\operatorname{SSP}} \mathbf{1}(0+1) \\
\underline{\text { Verts }} \mathbf{1}(0+1) \\
\underline{\text { Indep }} \mathbf{1}(1+0)\end{array}$ & $\begin{array}{l}\text { SSP } 6(0+6) \\
\text { Verts } 7(0+7) \\
\text { Autres } 4(2+2)\end{array}$ & $\begin{array}{l}\underline{\text { Verts } 2(0+2)} \\
\text { Indep } 1(0+1)\end{array}$ \\
\hline \multirow{5}{*}{$\begin{array}{l}\text { GALLES } \\
60 \text { sièges } \\
= \\
40 \text { FPTP } \\
+20 \text { listes régionales }\end{array}$} & Labour & $28(27+1)$ & $30(30+0)$ & $26(24+2)$ \\
\hline & Pl. Cym. & $17(9+8)$ & $12(5+7)$ & $15(7+8)$ \\
\hline & Cons & $9(1+8)$ & $11(1+10)$ & $12(5+7)$ \\
\hline & Lib-Dems & $6(3+3)$ & $6(3+3)$ & $6(3+3)$ \\
\hline & Autres & 0 & $1(1+0)$ & Indep $1(1+0)$ \\
\hline
\end{tabular}

\begin{tabular}{|l|l|l|l|}
\hline & & 2011 & 2016 \\
\hline $\begin{array}{l}\text { ECOSSE } \\
129 \text { sièges } \\
=\end{array}$ & Labour & $37(15+22)$ & $24(3+21)$ \\
\cline { 2 - 4 } & SNP & $69(53+16)$ & $63(59+4)$ \\
\hline
\end{tabular}

73 FPTP 


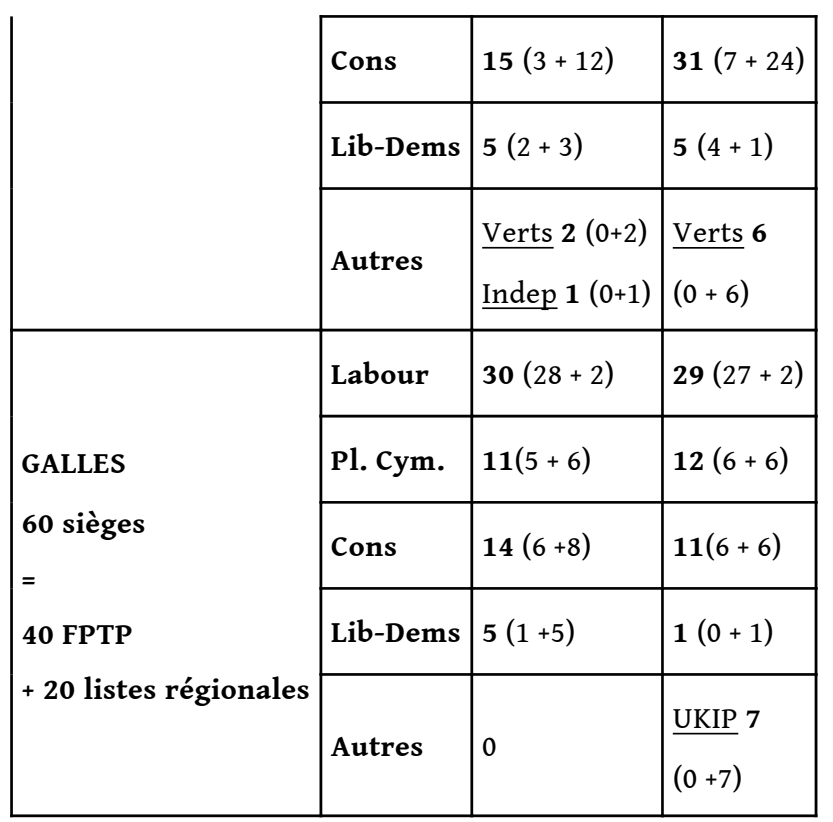

NOMBRE DE SIÈgES À POURVOIR: SCOTTISH PARLIAMENT: 129 MEMBERS OF PARLIAMENT (MSPS) = 73 $($ FPTP $=\mathbf{5 7} \%)+56$ (REgIONAL LISTS $=43 \%$ ); NATIONAL ASSEMBLY FOR WALES: 60 ASSEMBLY MEMBERS $($ AMS $)=40($ FPTP $=66 \%)+20($ REgIONAL LISTS $=33 \%)$ SSP: SCOTTISH SOCIALIST PARTY.

9 Au pays de Galles la situation était en tous points comparable, la hiérarchie partisane identique et les scores obtenus par les quatre principaux partis (travaillistes, nationalistes, conservateurs, libéraux-démocrates) apparaissaient -de façon extrêmement frappante- pratiquement similaires (cf. tableaux 1 \& 2).

10 Tout comme en Ecosse, le système partisan s'organisait autour d'un quadripôle, dominé par le Labour et dont les nationalistes de Plaid Cymru constituaient la principale force d'opposition, conservateurs et libéraux-démocrates étant en concurrence directe pour le rôle de troisième force. Par ailleurs le leadership travailliste se trouvait limité par le scrutin proportionnel, qui empêchait le Labour d'avoir la majorité absolue au Senedd (28 sièges sur 60), ce qui allait l'entrainer dans un gouvernement de coalition avec les libéraux-démocrates à partir de 2000 jusqu'en 2003. C'est ce même scrutin proportionnel qui assurait aux conservateurs leur représentation à l'Assemblée galloise (8 sièges sur 9) et procurait une assise certaine aux nationalistes gallois -de façon moins décisive toutefois par rapport à la situation écossaise- sans toutefois permettre aux petites formations (écologistes notamment) d'avoir des élus.

11 Dans les deux nations périphériques, le centre de gravité idéologique se situait clairement au centre gauche de l'échiquier politique, travaillistes, nationalistes et libérauxdémocrates partageant un même agenda social-démocrate.

12 Ce premier scrutin aux assemblées territoriales permettait également de mesurer l'impact de la dévolution sur l'évolution du système partisan par rapport à la précédente consultation, à savoir les élections législatives britanniques tenues deux ans auparavant (voir tableau 3).

13 Tableau 3 : Comparaison des résultats entre les élections législatives 1997 et les élections territoriales 1999 en Ecosse et au pays de Galles (\% des voix obtenues) 


\begin{tabular}{|l|l|l|l|l|}
\hline & \multicolumn{2}{|l|}{ ECOSSE } & \multicolumn{2}{l|}{ GALLES } \\
\hline & 1997 Westminster & $\begin{array}{l}1999 \\
\text { Holyrood }\end{array}$ & $\begin{array}{l}1997 \\
\text { Westminter }\end{array}$ & $\begin{array}{l}1999 \\
\text { Senedd }\end{array}$ \\
\hline SNP / PC & 22,1 & 28,7 & 9,9 & 28,4 \\
\hline Labour & 45,6 & 38,8 & 54,7 & 37,6 \\
\hline Conservateurs & 17,5 & 15,6 & 19,6 & 15,8 \\
\hline Lib-Dems & 13 & 14,2 & 12,4 & 13,5 \\
\hline Autres & 1,9 & 2,7 & 3,4 & 4,7 \\
\hline
\end{tabular}

*Score de 1999 : il s'agit du premier scrutin de circonscription uninominal majoritaire à un tour (FPTP) nationalistes tiraient de la situation était évident, les nouvelles assemblées décentralisées apparaissant comme un cadre propice à l'expression de leurs revendications. C'était particulièrement vrai au pays de Galles, où Plaid Cymru profitait des élections de 1999 pour faire un bond électoral spectaculaire (+ 18 points par rapport aux législatives de 1997), réalisant son meilleur score absolu pour une consultation régulière.

D'une façon générale, les deux principales victimes de ce nouveau scrutin étaient d'une part les conservateurs et d'autre part les travaillistes -notamment au pays de Galles, où leur chute (-17 points) était du même ordre de grandeur que la progression nationalistesoit les deux formations britanniques de gouvernement alternativement au pouvoir à Londres.

\section{3 : stabilisation du modèle et affirmation du multipartisme}

Par rapport à cette situation initiale de convergence (quasi-) absolue, la deuxième élection aux assemblées décentralisées de mai 2003 a apporté quelques correctifs notables, sans que l'architecture globale des systèmes partisans territorialisés ne soit remise en cause (cf. tableaux $1 \& 2$ ).

Ainsi les travaillistes continuaient-ils de dominer les scènes écossaise et galloise, tandis que les nationalistes du SNP et $P C$ conservaient leur rôle de challenger majeur. Ces derniers étaient cependant assez nettement en retrait en Ecosse comme au pays de Galles, tandis que le Labour connaissait des situations contrastées, déclinant légèrement en Ecosse tout en progressant modestement au pays de Galles. Donc si l'écart entre travaillistes et nationalistes demeurait à peu près similaire en Ecosse, il se creusait au pays de Galles. D'autre part les scores des conservateurs et des libéraux-démocrates étaient globalement stables, les deux formations étant toujours en concurrence pour la troisième place, avec un avantage pour les premiers -notamment au pays de Galles- qui 
plus que jamais continuaient d'être les principaux bénéficiaires du deuxième vote à la proportionnelle.

Dans ce paysage électoral reproduit globalement à l'identique par rapport au précédent scrutin, une différence cependant émergeait, sous la forme d'une certaine fragmentation de la représentation. Ainsi au pays de Galles le parti anti-européen UKIP s'insérait dans la compétition électorale, réalisant un score similaire aux écologistes (7\% à eux deux) tandis que pour la première fois un siège à l'assemblée galloise était attribué à une formation n'appartenant pas aux quatre principaux partis. Le Labour détenait cependant autant de sièges que les autres partis réunis, ce qui lui permit de gouverner sans aucun apport extérieur pendant la mandature.

C'est en Ecosse que la diversification de la représentation apparaissait de façon frappante, débouchant sur le «Rainbow Parliament ». Avec près de $14 \%$ des voix cumulées pour les écologistes et les socialistes radicaux, 7 partis différents siégeant à Holyrood et 17 sur 129 des sièges (soit 13\%) occupés par des élus extérieurs au quadripôle dominant voire « hors système ", l'Ecosse semblait s'orienter vers un multipartisme proche du modèle scandinave et plus conforme aux potentialités du type de scrutin choisi. Ce relatif éclatement de la représentation politique allait de pair avec l'absence d'un parti clairement majoritaire, forçant le Labour à poursuivre le principe du gouvernement de coalition avec les Libéraux-démocrates en Ecosse sur la durée de la législature (2003-2007).

\section{7 : des changements importants mais divergents en Ecosse et au pays de Galles}

Contrairement au précédent scrutin, la troisième élection de mai 2007 a reflété des changements importants et amorcé un net processus de divergence entre les situations écossaise et galloise (cf. tableaux $1 \& 2$ ).

21 En Ecosse le scrutin a été marqué par la progression spectaculaire du Scottish National Party, qui fit jeu égal avec les travaillistes au scrutin majoritaire -gagnant 9 points par rapport à 2003- et devança légèrement ces derniers au scrutin proportionnel (grâce à une progression de 10 points par rapport à 2003). Pour un siège d'écart et grâce à la représentation procurée par le vote à la proportionnelle, le SNP devenait pour la première fois le premier parti écossais à Holyrood, et pouvait former un gouvernement nationaliste minoritaire (soutenu par les Verts). Les conservateurs comme les libéraux-démocrates étaient toujours à des niveaux de popularité comparables mais désormais loin derrière, ayant récolté deux fois moins de suffrages que les nationalistes et les travaillistes. Par ailleurs la diversification de la représentation écossaise amorcée en 2003 ne se confirmait pas, les écologistes étant les seuls à maintenir une représentation au Parlement écossais mais à un niveau nettement moindre.

22 La scène écossaise apparaissait à nouveau dominée par un quadripôle partisan, désormais constitué de deux duopoles, avec un couple majoritaire SNP/Labour et un couple minoritaire Conservateurs/Libéraux-Démocrates.

23 Au pays de Galles le parti travailliste régressait nettement par rapport à la précédente consultation (-8 et -7 points aux différents scrutins), plus encore qu'en Ecosse. Cependant contrairement à la situation écossaise, cette baisse du Labour ne profitait que marginalement aux nationalistes de Plaid Cymru mais faisait le bonheur des conservateurs 
-à égalité avec $P C$ pour la deuxième place- les libéraux-démocrates étant désormais largement distancés. Si le Labour obtenait exactement le même résultat en Ecosse et au pays de Galles, la division de l'opposition en deux formations globalement équivalentes (nationalistes et conservateurs) permettait aux travaillistes gallois de conserver leur leadership, contrairement à la situation écossaise, et de continuer à gouverner, d'abord en situation minoritaire puis en partenariat avec Plaid Cymru. Par ailleurs si la fragmentation de la représentation amorcée lors des élections précédentes ne se confirmait pas au pays de Galles, les écologistes et UKIP étaient toujours présents au deuxième scrutin et désormais rejoints par le British National Party, signe d'une certaine droitisation de la scène politique galloise, un autre élément de divergence avec l'Ecosse.

\section{1 : la confirmation de la divergence entre les deux modèles}

Cette divergence entre les deux situations observables en Ecosse et au pays de Galles va se confirmer et s'accentuer à l'occasion des élections territoriales de mai 2011 (voir tableaux $1 \& 2)$.

Dans un contexte politique inédit marqué à la fois par la présence d'un gouvernement de coalition dominé par les Conservateurs à Londres (à partir de 2010) et d'une administration nationaliste aux commandes à Edimbourg depuis quatre ans, les résultats au Parlement écossais ont confirmé la popularité du SNP. Ce dernier progressait à nouveau de façon extrêmement spectaculaire (+ 13 points par rapport à 2007) au point de recueillir la majorité absolue des sièges au Parlement de Holyrood. Ce résultat, qui était difficilement envisageable au vu du mode de scrutin et jamais atteint par aucun parti jusqu'à lors, allait permettre au SNP de gouverner l'Ecosse en situation majoritaire de 2011 à 2016. Les travaillistes, à nouveau en léger recul, étaient désormais supplantés comme parti dominant et confirmés dans leur statut d'opposant principal aux nationalistes, dont la gestion des affaires écossaises avait assis la popularité sur la scène locale. On assistait là clairement à une inversion des rôles par rapport à la première phase de la dévolution (1999-2007). Par ailleurs la domination du duopole SNP/Labour sur la scène écossaise était à son zénith : les deux partis récoltaient à eux deux plus des troisquarts de suffrages au scrutin majoritaire, tandis que les conservateurs semblaient plafonner à un niveau modeste et que les libéraux-démocrates s'effondraient et perdaient la moitié de leurs soutiens, pâtissant de leur participation au gouvernement londonien aux yeux de l'opinion écossaise.

Au pays de Galles, par contre, la situation était toute autre. En effet le scrutin de mai 2011 voyait les travaillistes accentuer leur emprise sur la scène galloise pour obtenir un niveau de popularité record depuis l'instauration de la dévolution. Un autre fait marquant fut la progression des conservateurs qui doublèrent les nationalistes de $P C$ pour devenir la première force d'opposition au gouvernement travailliste minoritaire, tandis que les libéraux-démocrates subissaient, tout comme en Ecosse, un net recul. 


\section{6 ou l'accentuation de la divergence entre les situations écossaise et galloise} sans toutefois perdre leur leadership sur la scène électorale locale. Les nationalistes de $P C$ -en légère progression- et les conservateurs -en baisse- ont obtenu des résultats comparables et demeurent en compétition pour le rôle de principale alternative au Labour , qui continue de tirer avantage du mode de scrutin FPTP. Tout comme en Ecosse, les libéraux-démocrates ont vu leur déclin électoral se poursuivre et leur représentation au Senedd est désormais réduite à un seul élu. Dans le même temps cette élection a été marquée par la progression spectaculaire de UKIP, qui obtient un résultat à deux chiffres aux deux votes et entre à l'assemblée galloise avec une représentation respectable (7 sièges sur 60), tandis qu'une autre formation de type populiste et prônant l'abolition de l'assemblée galloise, rencontrait un certain écho dans l'opinion'

\section{La compétition partisane post-dévolution : multipartisme limité et système à parti dominant}

31 Si en 1999 les systèmes partisans territorialisés des deux nations périphériques étaient structurés de façon en tous points semblable, la situation apparait bien différente aujourd'hui.

En Ecosse la compétition électorale au Parlement de Holyrood s'organise désormais autour d'un système à cinq éléments, constitué par un parti dominant (le SNP), deux forces d'opposition de poids comparable (travaillistes et conservateurs) et deux formations de second ordre (écologistes et libéraux-démocrates).

Revue Française de Civilisation Britannique, XXII-4 | 2017 

scrutin à la proportionnelle- permis d'installer sur la durée une cinquième force -les Verts- tandis que la hiérarchie partisane s'est trouvée totalement bouleversée. En effet si en 1999 les travaillistes dominaient largement la scène politique écossaise, suivis à distance par le Scottish National Party, puis les conservateurs et les libéraux-démocrates, ce sont aujourd'hui les nationalistes qui écrasent la compétition électorale, devançant nettement conservateurs et travaillistes désormais au coude à coude, tandis que les libéraux-démocrates ont connu un déclin régulier pour se retrouver aujourd'hui marginalisés. quinzaine de points et la perte de plus de la moitié de leur représentation à Holyrood) est allé de pair avec l'affirmation parallèle et tout aussi spectaculaire des nationalistes: désormais quasi-hégémonique au scrutin FPTP, le SNP a tiré un profit maximum de ces élections territorialisées pour supplanter le Labour comme parti dominant et s'affirmer comme l'élément central du jeu politique écossais. Les conservateurs, initialement hostiles à la dévolution et par ailleurs opposés par principe au scrutin à la proportionnelle, ont réussi à établir une représentation durable au sein du Parlement écossais, puis à s'affirmer comme un élément d'opposition grâce à ce deuxième vote à la proportionnelle mis en place dans le cadre de la décentralisation institutionnelle. également multipartite, et structuré autour de cinq formations principales. Un peu plus diversifié qu'en Ecosse, il est organisé avec un parti dominant (le Labour), deux formations d'opposition d'importance comparable (nationalistes de Plaid Cymru et conservateurs), une nouvelle force émergente (UKIP) et plusieurs partis de faible importance dont les libéraux-démocrates, qui ont suivi la même pente déclinante qu'en Ecosse.

ar rapport à cette dernière, le système apparait beaucoup plus stable sur la durée : ainsi la hiérarchie interne aux trois principaux partis n'a pas évolué entre 1999 et 2016, et elle s'est reproduite pratiquement à l'identique à chaque scrutin. Par ailleurs les travaillistes, qui ont fait partie de tous les gouvernements gallois sans exception depuis 1999, se sont maintenus comme l'élément pivot de la scène politique locale. Les nationalistes, quant à eux, ne sont pas parvenus à tirer profit des élections à l'assemblée galloise pour progresser dans l'électorat : après un très bon score initial en 1999, Plaid Cymru a connu un résultat en retrait et stable sur la période, son influence plafonnant à environ un cinquième de l'électorat. Tout comme celle du SNP en Ecosse, la domination travailliste actuelle au pays de Galles est très solide, d'autant que l'opposition se compose de deux forces globalement équivalentes et entre lesquelles toute alliance ou coalition semble actuellement impossible.

Un autre élément de différenciation important entre les systèmes partisans écossais et gallois a trait à UKIP : totalement marginal en Ecosse, le parti populiste anti-européen s'est affirmé sur la scène galloise sur la période, quel que soit le type d'élections, et le mode de scrutin à la proportionnelle de l'assemblée galloise lui permet aujourd'hui d'avoir une représentation non négligeable au Senedd.

Le succès de UKIP - de même que la percée récente du parti réclamant l'abolition de l'assemblée galloise- introduit dans le système partisan gallois un élément populiste «hors système", qui le fait évoluer d'un pluralisme modéré vers un pluralisme plus polarisé6. Il va de pair avec une évolution du centre de gravité du système politique 
gallois vers la droite: ainsi le score cumulé du Labour et de $P C$ sur la période décline d'environ deux tiers à un peu plus de la moitié de l'électorat, tandis que celui additionné entre les Conservateurs et UKIP est globalement multiplié par deux.

En Ecosse, par contre, le résultat cumulé des travaillistes et des nationalistes est demeuré stable entre 1999 et 2016 - de l'ordre des deux-tiers des suffrages- tandis que l'extrême gauche a ponctuellement obtenu quelques sièges au Parlement et que les écologistes, situés également à gauche sur l'axe politique, ont maintenu une présence significative d'un scrutin à l'autre.

\section{Nationalisme et travaillisme en Ecosse et au pays de Galles : deux trajectoires différentes}

40 La divergence majeure entre les situations écossaise et galloise depuis la mise en route de la dévolution a trait aux évolutions des mouvements travailliste et nationaliste dans les deux nations périphériques.

En Ecosse, les nationalistes du SNP ont pu utiliser les élections décentralisées pour investir le parlement de Holyrood et s'établir comme une alternative crédible aux travaillistes au plan local. A partir du tournant de 2007, leur accession au pouvoir les a installés dans l'opinion comme une force gestionnaire efficace, alliant compétence, recherche du consensus et leadership. Dans le même temps leurs succès locaux ont permis aux nationalistes de remettre sur la table leur agenda institutionnel en faveur de l'indépendance, ce qui a débouché sur le référendum de septembre 2014. Le poids de ce dernier continue aujourd'hui de structurer la scène politique écossaise autour de l'enjeu de l'autonomie politique et du clivage entre nationalisme et unionisme : d'un côté, le SNP et les autres petites formations -dont les Verts- favorables à l'indépendance écossaise, de l'autre le camp unioniste, dont travaillistes et conservateurs -en opposition sur l'axe gauche-droite- se disputent le leadership.

En une quinzaine d'années et grâce à ce scrutin décentralisé, le SNP a supplanté le parti travailliste en Ecosse en s'imposant aux dépens de ce dernier sur deux terrains: idéologique d'une part, en se faisant le champion d'un progressisme de centre-gauche et de défense des acquis sociaux de l'Etat-Providence; institutionnel ensuite, en se posant comme le premier défenseur des intérêts écossais vis-à-vis de Londres et le mieux à même d'obtenir le degré d'autonomie maximal pour l'Ecosse par rapport au cadre britannique.

La situation galloise est très différente. Le scrutin décentralisé a contribué à assurer une visibilité certaine aux nationalistes, sans remettre en cause le leadership historique des travaillistes sur la scène politique locale. Contrairement à l'Ecosse où le SNP a profité du scrutin décentralisé pour proposer un message politique transversal interpellant toute la société écossaise et pour combattre frontalement le Labour notamment dans ses bastions électoraux ouvriers et urbains du Central Belt, Plaid Cymru a tenu un discours beaucoup plus ambigu sur l'enjeu constitutionnel et continué de centrer son programme sur la défense de la langue et de la culture galloises, ciblant en priorité une population -les électeurs galloisants- et un territoire -Y Fro Gymraeg- spécifiques. Par ailleurs plutôt que de se poser en alternative possible à la mainmise travailliste sur les institutions décentralisées, les nationalistes gallois ont fait le choix inverse notamment en s'associant en 2007 au Labour comme force d'appoint au sein d'un gouvernement de coalition. 
à la différence de leurs homologues écossais, ont aussi utilisé les nouvelles institutions décentralisées pour porter un message politique de type nationaliste, qui leur permet d'être toujours crédibles au sein de l'opinion pour être la « voix » du pays de Galles et de rester au point d'équilibre des deux enjeux majeurs du débat politique.

\section{Les clivages droite-gauche et nationalisme- unionisme : un impact différencié dans les deux nations périphériques}

Avec la dévolution les deux nations périphériques ont mis en place des systèmes partisans territorialisés originaux, associant des éléments relevant d'un pluralisme limité -mettant en compétition une demi-douzaine de formations- et d'un modèle à parti dominant. Si en Ecosse travaillistes et nationalistes se sont affrontés depuis 1999 pour le statut de formation pivot autour de laquelle se forme le gouvernement et se sont succédé au pouvoir, au pays de Galles le leadership du Labour n'a jamais été remis en question sur la période, et c'est autour de ce dernier que se sont organisées toutes les majorités sans exception.

D'autre part le débat politique s'est structuré autour des deux enjeux prioritaires identifiés en 1999, à savoir le clivage traditionnel gauche-droite et la relation centrepériphérie. Par rapport à cela le système partisan écossais reflète aujourd'hui une très forte polarisation autour de l'enjeu constitutionnel de l'autonomie/indépendance ${ }^{7}$, contrairement au modèle gallois où la polarisation sur l'enjeu territorial apparait beaucoup plus limitée. De fait la dimension socio-économique du débat partisan semble prédominante au pays de Galles, où UKIP apporte désormais une connotation antisystème inédite.

47 Après près de deux décennies de dévolution et cinq scrutins décentralisés successifs, le format et les résultats de la compétition partisane dans les nations périphériques ont notablement évolué, et les situations écossaise et galloise ont divergé sur de nombreux aspects. Cette différenciation progressive entre les modèles écossais et gallois depuis le premier scrutin territorial de 1999 s'illustre aussi si l'on met en parallèle la configuration actuelle de la compétition inter partisane issue des scrutins décentralisés de 2016 avec celle résultant des dernières élections législatives britanniques, tenues en 2015 (voir tableau 4).

Tableau 4 : Comparaison des résultats entre les élections législatives 2015 et les élections territoriales 2016 en Ecosse et au pays de Galles (\% de voix obtenues)

\begin{tabular}{|c|c|c|c|c|}
\hline & \multicolumn{2}{|l|}{ EcossE } & \multicolumn{2}{|l|}{ GALLES } \\
\hline & $\begin{array}{l}2015 \\
\text { Westminster }\end{array}$ & $\begin{array}{l}2016 \\
\text { Holyrood }\end{array}$ & $\begin{array}{l}2015 \\
\text { Westminster }\end{array}$ & $\begin{array}{l}2016 \\
\text { Senedd }\end{array}$ \\
\hline SNP / PC & 50,0 & 46,5 & 12,1 & 20,5 \\
\hline Labour & 24,3 & 22,6 & 36,9 & 34,7 \\
\hline
\end{tabular}




\begin{tabular}{|l|l|l|l|l|}
\hline Conservateurs & 14,9 & 22 & 27,2 & 21,1 \\
\hline Lib-Dems & 7,5 & 7,8 & 6,5 & 7,7 \\
\hline Verts & 1,3 & 0,6 & 2,6 & 2,5 \\
\hline UKIP & 1,6 & $/$ & 13,6 & 12,5 \\
\hline
\end{tabular}

*Score de 2016 : il s'agit du premier scrutin de circonscription uninominal majoritaire à un tour (FPTP)

\section{Elections décentralisées et élections britanniques : d'une compétition partisane à l'autre}

Si l'on s'en tient à une comparaison terme à terme portant uniquement sur les résultats du premier scrutin majoritaire (FPTP) aux deux types d'élections, on remarque que le modèle partisan pour les élections à Holyrood et au Senedd est aujourd'hui relativement proche de celui des élections à la chambre des Communes. Dans les deux cas, la hiérarchie partisane est identique, et les scores obtenus par les différentes formations politiques lors des deux types d'élections sont assez comparables. Cependant plusieurs différences notables sont à relever, qui concernent à la fois l'évolution générale par rapport à la situation des premières élections de 1997/99 (voir tableau 3) et la différence entre les cas écossais et gallois.

Concernant le pays de Galles, la configuration d'ensemble n'a guère évolué par rapport à la situation initiale (voir tableau 4): les deux principaux partis britanniques travaillistes et conservateurs - et notamment ces derniers- obtiennent toujours de meilleurs résultats aux élections législatives britanniques plutôt qu'à l'assemblée galloise, tandis que la problématique est inversée pour les nationalistes de Plaid Cymru, qui continuent logiquement de retirer un avantage important du scrutin territorial.

En Ecosse par contre la situation apparait aujourd'hui toute autre et, à certains égards, paradoxale. En effet si pour les travaillistes la situation n'a guère évolué par rapport aux débuts de la dévolution, dans la mesure où leur résultat est légèrement meilleur pour les élections à la chambre des Communes, pour les conservateurs en revanche c'est l'inverse qui est vrai: toujours nettement rejetés par l'opinion écossaise lors des élections législatives britanniques, ils parviennent à améliorer assez nettement leur score à l'occasion des élections à Holyrood, et ils apparaissent aujourd'hui comme les principaux bénéficiaires de la dévolution.

La deuxième différence importante concerne les nationalistes du Scottish National Party: contrairement à la situation écossaise initiale en vigueur en 1999 et aussi à la situation prévalant actuellement au pays de Galles, le SNP réalise aujourd'hui lors des élections législatives un score record, meilleur encore que celui obtenu au précédent scrutin de Holyrood. Les nationalistes écossais tirent ainsi le bénéfice de leur domination quasiabsolue dans le cadre du premier scrutin de circonscription, transférant à l'échelle de la représentation britannique leur suprématie acquise lors du scrutin territorial de 2011.

Ce résultat spectaculaire du SNP aux élections législatives britanniques et son leadership inédit sur la représentation écossaise à Wesminster illustre une nouvelle fois la prégnance du clivage nationalisme/unionisme dans le débat politique écossais, ainsi que 
la force de la polarisation de l'électorat autour de l'enjeu constitutionnel. Il semble indiquer l'ouverture d'une troisième phase originale de la compétition inter-partisane post-dévolution dans la périphérie britannique.

\section{BIBLIOGRAPHIE}

BENNIE, Lynn \& CLARK, Alistair, « Towards Moderate Pluralism: Scotland's Post Devolution Party System, 1999-2002 », British Elections \& Parties Review, 13, 2003, p. 134-155.

BOHRER, Robert E. \& KRUTZ, Glen S., « The Devolved Party Systems of the United Kingdom, Subnational Variations from the National Model », Party Politics, 11/6, 2005, London: Sage, p. 654-673. HASSAN, Gerry (dir.), The Modern SNP, From Protest to Power, Edinburgh, Edinburgh University Press, 2009.

HOUGH, Dan \& JEFFREY, Charley (dir.), Devolution and Electoral Politics, Manchester, Manchester University Press, 2006

INGRAM, Robert, First or Second Order? « Will the Scottish and Welsh Elections Deliver Devolution? », British Elections \& Parties Review, 13, 2003, p. 107-133.

LEYDIER, Gilles, « Mobilisation (record) et polarisation (spectaculaire) de l'électorat écossais autour de l'enjeu de l'indépendance ", Le référendum sur l'indépendance en Ecosse, Revue française de civilisation britannique, Université Paris III Sorbonne Nouvelle, Paris, vol. XX n², 2015.

LEYDIER, Gilles, « A surge of nationalism ? Les ressorts de l'hégémonie politique du SNP ». Colloque international Enjeux et perspectives du référendum écossais pour le Royaume-Uni et pour l'Europe, Université de Nantes, septembre 2015.

LEYDIER, Gilles, « It was not supposed to happen : une mise en perspective historique des élections au Parlement écossais », Moya Jones \& Edwige Camp-Piétrain (dir.) Les consultations électorales du 5 mai 2011 au Royaume-Uni, revue Textes \& Contextes, Université de Bourgogne, 2013.

LYNCH, Philip, « Party System Change in Britain: Multi-party Politics in a Multi-Level Polity », British Politics, 2/3, p. 323-346, 2007.

Mc ALLISTER, Laura, Plaid Cymru, The Emergence of a Political Party, Glasgow, Bell \& Bain, 2001.

MAIR, Peter, Party System Change: Approaches and Interpretations, Oxford, Oxford University Press, 1997.

MASSETTI, Emanuele, The Scottish and Welsh Party Systems Ten Years after Devolution: Format, Ideological Polarization and Structure of Competition, University of Sussex, 2008.

SARTORI, Giovanni, Parties and Party Systems: A Framework for Analysis, Cambridge, Cambridge University Press, 1976.

WYN JONES, Richard \& SCULLY, Roger, « Devolution and Electoral Politics in Scotland and Wales ", Publius: The Journal of Federalism, 36/1, 2006, p. 115-134. 


\section{NOTES}

1. Depuis 1999, cinq scrutins décentralisés se sont tenus au même moment en Ecosse et au pays de Galles, respectivement en 1999, 2003, 2007, 2011 et 2016 (ce dernier scrutin ayant été décalé d'un an afin de ne pas coïncider avec les élections législatives britanniques du printemps 2015).

2. On reprendra la terminologie de Giovanni Sartori, définissant le système partisan ('party system') comme 'the system of interactions resulting from inter-party competition'. SARTORI, Giovanni, Parties and Party Systems: A Framework for Analysis, Cambridge, Cambridge University Press, 1976, p. 42.

3. Additional Member System. Dans une élection de type AMS chaque électeur possède deux votes, un premier pour choisir un candidat dans sa circonscription électorale, un deuxième pour sélectionner une liste de candidats présentés par les différentes formations politiques, au sein de régions territoriales regroupant plusieurs circonscriptions.

4. Ecosse et pays de Galles confondus.

5. Il s'agit de l'organisation Abolish Welsh Assembly.

6. Selon la typologie partisane de Giovanni Sartori (1976).

7. Voir LEYDIER Gilles, "Mobilisation (record) et polarisation (spectaculaire) de l'électorat écossais autour de l'enjeu de l'indépendance ", Le référendum sur l'indépendance en Ecosse, Revue française de civilisation britannique, Université Paris III Sorbonne Nouvelle, Paris, vol. XX n², 2015.

\section{RÉSUMÉS}

L'objet de cet article est d'analyser l'évolution de la compétition entre les partis politiques dans la périphérie de la Grande Bretagne depuis la mise en place de la dévolution, à travers l'étude des résultats des élections aux assemblées décentralisées. La comparaison entre les situations en Ecosse et au pays de Galles fait ressortir une similitude totale à l'occasion des premières élections territoriales de 1999, puis la convergence grandissante entre les deux systèmes partisans au fil des différentes consultations. L'article insiste sur l'impact différencié des deux clivages gauchedroite et nationalisme-unionisme sur l'évolution politique contrastée des deux nations périphériques, ainsi que sur l'articulation particulière existante entre ce scrutin décentralisé et les élections législatives britanniques dans le contexte de la dévolution.

The aim of this article is to analyse the evolution of the party-system and electoral competition in the periphery of Great Britain since the beginning of the devolution process and the first decentralized elections in 1999. The comparison between the Scottish and Welsh cases highlights the complete convergence between the two situations in 1999 and their gradual divergence since then. The article insists on the differential impact of the right/left as well as nationalist/unionist cleavages upon the evolution of the local situation, and questions the specific type of relationships which has taken place between the parliamentary elections and the general ones in the two peripheral nations in the devolution context. 
INDEX

Mots-clés : Ecosse, Pays de Galles, élections décentralisées, système partisan, dévolution, nationalisme

\section{AUTEUR}

GILLES LEYDIER

Laboratoire Babel, EA 2649, Université de Toulon, 83041 Toulon 